\title{
Microfluidic in vitro brain endothelial monolayer model to evaluate cell-penetrating peptides
}

\author{
Bohye Chung ${ }^{1}$, Jaehoon Kim², Hui-Wen Liu², Jiyoung Nam³ ${ }^{3}$ Hyunho Kim², Hyun Jeong Oh², Yong Ho Kim \\ and Seok Chung ${ }^{1,2^{*}}$
}

\begin{abstract}
Blood vessels in central nervous system act as a great hurdle for drug delivery to the human brain. They only allow passage of water, some gases, lipid molecules, glucose and amino acid by selective transporter while restricting most of solutes and pathogens to protect brain. A lot of studies have tried to overcome this hurdle by discovering and optimizing brain deliverable drugs however the platforms used for preclinical stage are still limited. In this study, we constructed an in vitro 3-dimensional model for brain endothelial monolayer using hydrogel incorporated microfluidic device that provides an 3D extracellular matrix scaffold. We confirmed the stable endothelial barrier by staining a tight junction marker, VE-Cadherin, and strong block ability by comparing permeability with normal endothelial cells. Also, we succeed in verifying the strong permeability of angiopep-2 using our device that is known as a brain permeable peptide by utilizing receptor-mediated transcytosis. We propose our microfluidic device as an in vitro platform for evaluating various brain drugs or drug carrier candidates.
\end{abstract}

Keywords: Blood-brain barrier (혈뇌장 벽), Cell-penetrating peptide (세포 투과 펩타 이드), Microfluidics (미세유 체)

\section{Background}

Homeostasis controlling and protecting the brain from toxins and pathogens are very important for proper neuronal function and therefore the micro-vessels in brain have unique properties by forming a strong barrier structure called as blood-brain barrier (BBB) [1]. Brain endothelial cells (bECs) in BBB are in contact with pericytes and end-feet of astrocytes in basal lamina [2]. However, the roles of pericytes and astrocytes are known to support the barrier function of bECs and it is known that the properties of $\mathrm{BBB}$ mostly depend on the barrier structure of bECs formed by various junctions such as adherence junctions, tight junctions and gap junctions. Because of the crucial role of bECs for the barrier function of $\mathrm{BBB}$, they are considered as a determining $\mathrm{BBB}$ component for constructing in vitro BBB model [3].

\footnotetext{
*Correspondence: sidchung@korea.ac.kr

${ }^{1}$ KU-KIST Graduate School of Converging Science and Technology, Korea

University, Seoul, South Korea

Full list of author information is available at the end of the article
}

Because bECs strictly restrict the passage of molecules, permeability analysis for central nervous system (CNS) drugs is a big challenge. Most studies for evaluating molecule penetration through 2-dimensionally (2D) formed bEC monolayer have limitations, mainly in basal side components and non-precise quantification [4]. Microfluidic techniques might supply helpful solutions due to their clear vision and precise controllability on molecule transport [5]. We proved the reconstituted bEC barrier function comparing with normal endothelial cells and evaluated the cell-penetrating peptide, Angiopep-2 (targeting low density lipoprotein receptor-related protein 1 (LRP 1) to penetrate bEC monolayer and enhance drug delivery to brain [6]) with quantifying techniques using our device. Herein, we proposed a microfluidic model for brain endothelial barrier that can be utilized an evaluation platform for permeable neurotherapeutic drugs or drug carriers. 


\section{Methods}

The microfluidic chip has four gel channels and three medium channels (Fig. 1a). It was prepared by conventional soft lithography with SU-8-100 photoresist (MicroChem, USA) patterned silicon wafer (120-130 $\mu$ m thick) and cured PDMS at $80{ }^{\circ} \mathrm{C}$ for $1 \mathrm{~h}$. The cured PDMS was punched by biopsy punch and blunt needle and a glass coverslip was bonded to the imprinted PDMS using oxygen plasma treatment [7].

The poly-D-lysine (PDL; MW: 30,000-70,000; SigmaAldrich, USA) solution in distilled deionized water (DDW) was injected to coat channel surface to enhance adhesion between channel surface and hydrogel. Injected PDL solution was cured in humidified chamber at $37^{\circ} \mathrm{C}$ for $4 \mathrm{~h}$. Device was then washed by DDW and dried at $80{ }^{\circ} \mathrm{C}$ for $24 \mathrm{~h}$. Before cell seeding, type 1 collagen solution adjusted with a $0.5 \mathrm{~N} \mathrm{NaOH}$ solution to $\mathrm{pH} 7.4$ was incorporated in gel channel and gelled. Basement membrane (BM) solution, mixed by Matrigel and basal media, was then treated on the cell seeding surface of the incorporated hydrogel. 1.5 million cells/ml of hCMEC/ D3, human cerebral microvascular endothelial cell line, (for bECs) or hMVEC, human microvascular endothelial cells, (for control) were seeded on the recombinant BM coated hydrogel and cultured for 4 days (Fig. 1b).
When the bECs were sufficient confluent and formed bEC monolayer, $25 \mathrm{uM}$ FITC-dextran (MW: $3 \mathrm{kDa}$ ) for control or FITC conjugated Angiopep-2 (MW: $3 \mathrm{kDa}$ ) for test was injected into the bEC monolayer channel. To verify bEC permeability, fluorescent images were captured in 15 min and intensity was measured using Image J software (Fig. 1c). Diffusive permeability was calculated using Fick's first law as following:

$$
P_{D}=\frac{1}{\Delta C} \frac{d C}{d x} \mathrm{D}
$$

where, $P$ is diffusive permeability, $\Delta C$ intensity difference across bEC monolayer, $d C / d x$ intensity slope in gel and $\mathrm{D}$ diffusion coefficient.

Cells after experiments were fixed with $4 \%$ paraformaldehyde for $15 \mathrm{~min}$ at room temperature, and permeabilized with $0.1 \%$ Triton-X100 for $10 \mathrm{~min}$. 20\% Block Ace was added for blocking for $1 \mathrm{~h}$. Primary antibodies were treated overnight, and Alexa Fluor 488-conjugated secondary antibody was treated for $1 \mathrm{~h}$ at room temperature. Cell nuclei and actin filaments were counterstained with DAPI and rhodamine phalloidin, respectively. We got the stained images using a confocal microscope. a

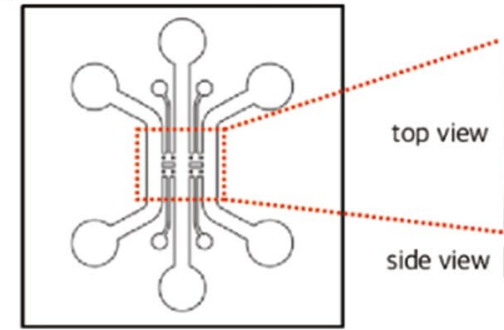

b

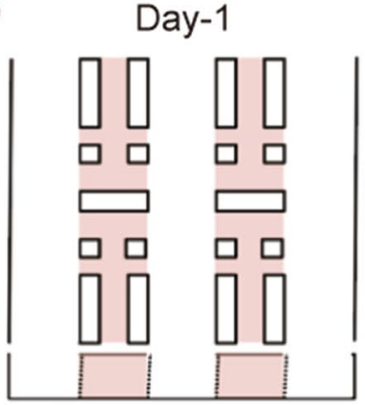

Hydrogel filling

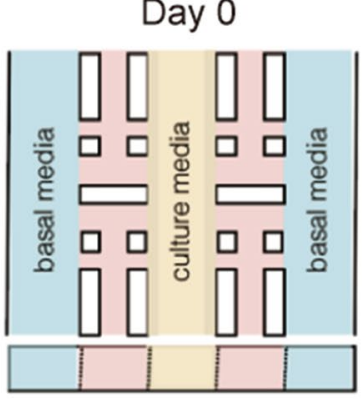

Media filling

\& BM deposition

C
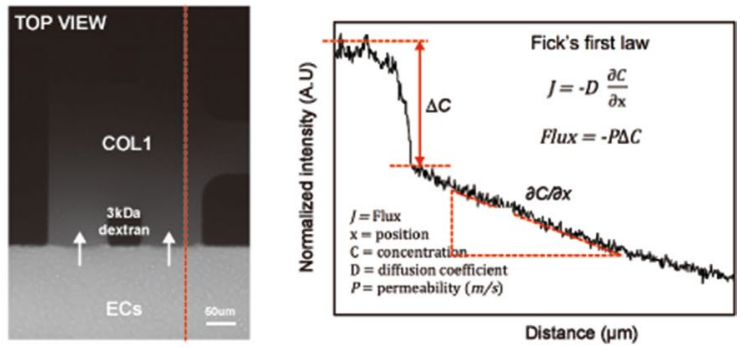

Day 4

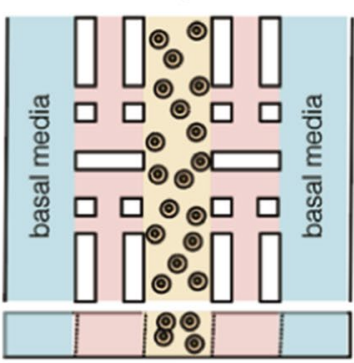

bECs seeding

Fig. 1 Schematics of microfluidic device design and permeability assay. Schematic overview of microfluidic chip at top and side view (a) and the preparation procedures for brain endothelial monolayer model in the microfluidic device (b) were shown. c Represents fluorescence image after injecting dextran into the device and intensity profile for permeability quantification 


\section{Results and discussions}

We tried to construct a stable brain endothelial barrier in our previously developed microfluidic device [8]. The bECs were seeded in the microfluidic chip and proliferated along the collagen wall, and finally we confirmed a 3D monolayer in day 4 (Fig. 2a). Moreover, the bECs consisting the monolayer showed a significant expression of junction protein, VE-cadherin, that is a major property of in vivo brain endothelial barrier (Fig. 2b). We also tried to prove that bECs have a better barrier function than normal ECs because it is known that the bECs have a few pinocytotic vesicles and low permeability in passing molecules [9]. We compared $3 \mathrm{kDa}$ FITC-dextran permeability and block/leakage ratio of hCMEC/D3 (bECs) with hMVEC (ECs) under same conditions. The result graph showed lower permeability and higher block/leakage ratio of bECs than normal ECs suggesting that bECs can construct a tighter monolayer with less leakage than normal ECs, as reported (Fig. 3a, b). Furthermore, we tested the cell-penetrating peptide, angiopep-2, using our device which is known as a BBB permeable peptide because it can interacts with LRP 1 that it is overexpressed in bECs [10]. We found the dramatically higher permeability of angiopep-2 than control case, same sized FITC-dextran and

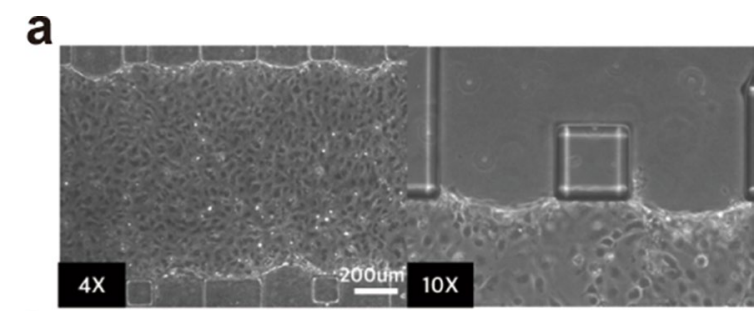

b

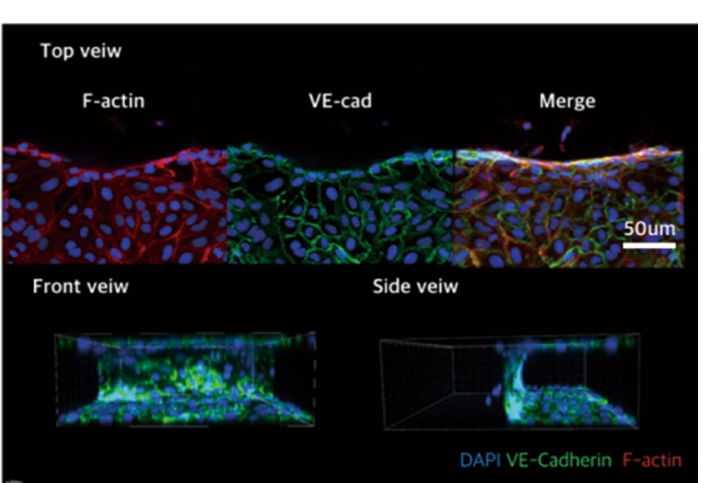

Fig. 2 The result images of microfluidic brain endothelial monolayer model at day 4. Phase images of brain endothelial cells in the microfluidic device at the bottom view (a) and the fluorescence images of them at the top, front and side view (b) were shown. The nucleus (blue), F-actin (red) and VE-cadherin (green) of brain endothelial cells in the microfluidic device was stained (scale bar $=50 \mu \mathrm{m})$

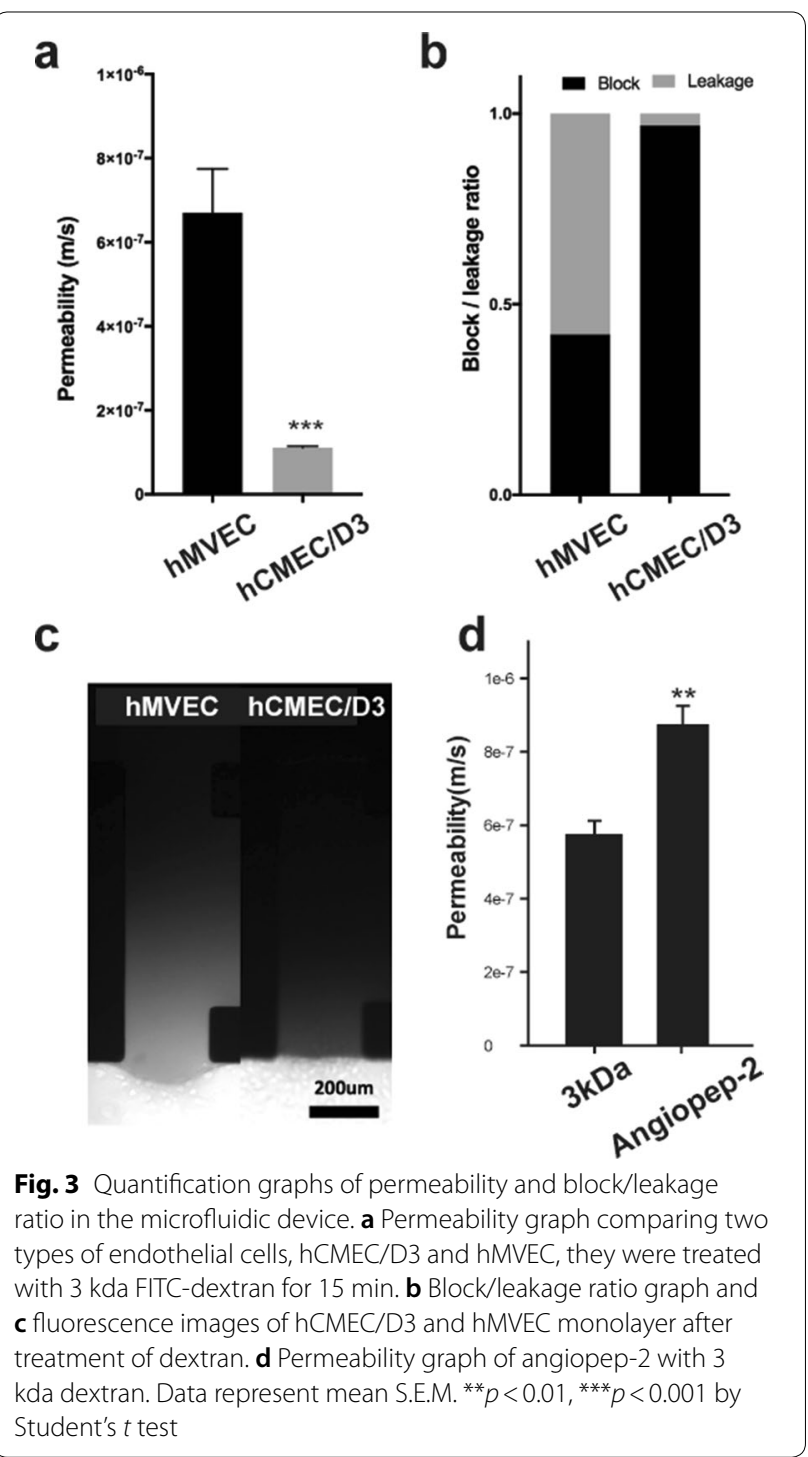

it suggested that angiopep- 2 can be penetrated to brain more easily than same sized molecules because of the interaction with LRP 1 in the surface of bECs (Fig. 3d). The result using our device showed the possibility of use as a brain drug carrier of angiopep-2. Our platform is promising as it can be used for comparing the permeability of various brain drug carriers in a quantitative manner. Therefore, we expect that our device will contribute to the early stage of brain permeable drug development.

\section{Authors' contributions}

SC devised the idea and supervised the project. JHK, HWL and HHK assisted the analysis and device fabrication. JYN fabricated experimental agents. $\mathrm{HJO}$ and YHK proposed the analytical method. $\mathrm{BHC}$ performed the analysis and wrote the overall manuscript. All authors read and approved the final manuscript. 


\section{Funding}

This work was supported by the National Research Foundation of Korea (NRF) Grant funded by the Korean government (MSIP: Ministry of Science, ICT and Future Planning) (No. 2017M2A2A7A02019899)

\section{Availability of data and materials}

Not applicable.

\section{Ethics approval and consent to participate}

Not applicable.

\section{Competing interests}

The authors declare that they have no competing interests.

\section{Author details}

${ }^{1}$ KU-KIST Graduate School of Converging Science and Technology, Korea University, Seoul, South Korea. ${ }^{2}$ School of Mechanical Engineering, Korea University, Seoul, South Korea. ${ }^{3}$ SKKU Advanced Institute of Nano Technology (SAINT), Sungkyunkwan University (SKKU), Suwon 16419, Republic of Korea.

Received: 21 June 2019 Accepted: 29 August 2019

Published online: 17 September 2019

\section{References}

1. Augustin HG, Koh GY (2017) Organotypic vasculature: From descriptive heterogeneity to functional pathophysiology. Science. https://doi. org/10.1126/science.aal2379

2. Cm de Lange $\mathrm{E}$ (2012) The physiological characteristics and transcytosis mechanisms of the blood-brain barrier (BBB). Curr Pharm Biotechnol 13:2319-2327
3. Luissint AC, Artus C, Glacial F, Ganeshamoorthy K, Couraud PO (2012) Tight junctions at the blood brain barrier: physiological architecture and disease-associated dysregulation. Fluids Barriers CNS 9:1-12

4. Nakagawa S, Deli MA, Kawaguchi H, Shimizudani T, Shimono T, Kittel Á, Tanaka K, Niwa M (2009) A new blood-brain barrier model using primary rat brain endothelial cells, pericytes and astrocytes. Neurochem Int 54:253-263

5. van der Helm MW, van der Meer AD, Eijkel JC, van den Berg A, Segerink LI (2016) Microfluidic organ-on-chip technology for blood-brain barrier research. Tissue Barriers 4(1):e1142493

6. Lajoie JM, Shusta EV (2014) Targeting receptor-mediated transport for delivery of biologics across the blood-brain barrier. Annu Rev Pharmacol Toxicol 55:613-631

7. Shin Y, Han S, Jeon JS, Yamamoto K, Zervantonakis IK, Sudo R, Kamm RD, Chung S (2012) Microfluidic assay for simultaneous culture of multiple cell types on surfaces or within hydrogels. Nat Protoc 7:1247-1259

8. Han S, Shin Y, Jeong HE, Jeon JS, Kamm RD, Huh D, Sohn LL, Chung S (2015) Constructive remodeling of a synthetic endothelial extracellular matrix. Sci Rep 5:1-10

9. Yu QJ, Tao H, Wang X, Li MC (2015) Argeting brain microvascular endothelial cells: a therapeutic approach to neuroprotection against stroke. Neural Regen Res 10:1882-1891

10. Nishijima T, Piriz J, Duflot S, Fernandez AM, Gaitan G, Gomez-Pinedo U, Verdugo JMG, Leroy F, Soya H, Nuñez A, Torres-Aleman I (2010) Neuronal activity drives localized blood-brain-barrier transport of serum insulin-like growth factor-1 into the CNS. Neuron 67:834-846

\section{Publisher's Note}

Springer Nature remains neutral with regard to jurisdictional claims in published maps and institutional affiliations.

\section{Submit your manuscript to a SpringerOpen ${ }^{\circ}$ journal and benefit from:}

- Convenient online submission

- Rigorous peer review

- Open access: articles freely available online

- High visibility within the field

- Retaining the copyright to your article

Submit your next manuscript at $\boldsymbol{\nabla}$ springeropen.com 